\title{
Reliability of LES in complex applications
}

\author{
Bernard J. Geurts
}

Multiscale Modeling and Simulation, Faculty EEMCS, University of Twente, P.O.Box 217, 7500 AE Enschede, The Netherlands

and

Anisotropic Turbulence, Fluid Dynamics Laboratory, Department of Applied Physics, Eindhoven University of Technology, P.O. Box 513, 5600 MB Eindhoven, The

Netherlands

\begin{abstract}
The accuracy of large-eddy simulations is limited, among others, by the quality of the subgrid parameterisation and the numerical contamination of the smaller retained flow-structures. We review the effects of discretisation and modelling errors from two different perspectives. First, we review a database-approach to assess the total simulation error and its numerical and modelling contributions. The interaction between the different sources of error in the kinetic energy is shown to lead to their partial cancellation. An 'optimal refinement strategy' for given subgrid model, given discretisation method and given flow conditions is identified, leading to minimal total simulation error at given computational cost. We provide full detail for homogeneous decaying turbulence in a 'Smagorinsky fluid'. The optimal refinement strategy is compared with the error-reduction that arises from grid-refinement of the dynamic eddy-viscosity model. Dynamic modelling yields significant error reduction upon grid refinement. However, at coarse resolutions high error-levels remain. To address this deficiency in eddyviscosity modelling, we then consider a new successive inverse polynomial interpolation procedure with which the optimal Smagorinsky constant may be efficiently approximated at any given resolution. The computational overhead of this optimisation procedure is well justified in view of the achieved reduction of the error-level relative to the 'no-model' and dynamic model predictions.
\end{abstract}

\section{$1 \quad$ Introduction}

Direct numerical simulation and large-eddy simulation are two important strategies for the numerical investigation of turbulent flows. Within the constraints of present-day computing infrastructure, the direct simulation approach is adopted for full resolution of flow-problems of modest complexity, e.g., to under-pin theoretical and modelling studies. Instead, the focus in large-eddy simulation is on a computationally more accessible coarsened flow description, which is obtained by low-pass spatial filtering (Geurts, 2003; Sagaut, 2001). This allows an external 
control over the required spatial resolution. However, low-pass filtering gives rise to the well-known closure problem for the turbulent stress, which represents the dynamic effects of the filtered-out small-scale turbulence on the retained flowstructures. Viewed entirely from the PDE-level corresponding to the spatially filtered Navier-Stokes equations, the remaining task is to close the system of equations by modelling these small-scale dynamic effects in terms of the resolved flow. Considerable effort has been put into construction, testing and tuning of such so-called subgrid models over the past years (see, e.g., Geurts, 2003; Meneveau, Katz, 2000; Lesieur, Metais, 1996; Sagaut, 2001). In such testing procedures one frequently compares predictions from large-eddy simulations to filtered data from direct numerical simulation and/or experimental data.

The above sketch of direct and large-eddy simulation is incomplete in at least one important respect, as it does not contain the unavoidable subsequent discretisation step. In fact, since the numerical representation in large-eddy simulations is typically associated with only marginal subgrid resolution, a significant alteration of the resolved scales' dynamics may be introduced in the computational model (Rogallo, Moin, 1984; Salvetti, Beux, 1998; Geurts, Froehlich, 2002; Meyers, Geurts, Baelmans, 2003).

A principal difficulty in comparing two turbulent solutions lies in their sensitivity to slight alterations in the initial conditions. Therefore, we consider DNS and LES that start from the same (filtered) initial condition, and restrict to decaying turbulence in this paper. By adhering to rather modest Reynolds numbers a comparison of the deterministic evolution of model turbulent flows is feasible. In these situations both DNS and LES are available. A judicious combination of these results allows arriving at an approximate decomposition of the total effect of the errors in terms of a discretization component and a modelling component. These effects may be of opposite signs (Vreman, Geurts, Kuerten, 1996) and counteract. This leads to significant resolution dependence of optimal model parameters that yield lowest total error at given resolution, e.g., measured as L2 norm. In particular, strong dependencies are observed in case the grid spacing is near the 'large-scale border' of an inertial range.

It is the purpose of this paper to review and quantify the numerical error dynamics explicitly. Next to the filter-width $\Delta$, the specification of the numerical method implies the introduction of a second length-scale $h$, which characterises the (local) computational grid spacing. Correspondingly, the discretisation step induces a second element of possible flow filtering (Geurts, Van der Bos, 2005). The difficulty hence resides in assessing the modelling and discretisation errors and their dynamic interaction in order to arrive at a specification of simulation and modelling parameters which are optimal, i.e., yield minimal total simulation error in the quantities of interest, at given computational cost. In this paper we review consequences of the interactions that occur between discretisation and subgrid modelling errors. Moreover, we propose a simple optimisation procedure to approximate optimal simulation parameters. 
The relative importance of the turbulent stresses compared to the numerically induced contributions depends strongly on the sub-filter resolution $r=\Delta / h$ (Ghosal, 1996). If $r$ is sufficiently large, the grid-independent large-eddy solution, consistent with the assumed value of $\Delta$ and the adopted subgrid model, may be accurately approximated. However, large-eddy simulation of applications with a realistic complexity is typically hampered by only marginal resolution corresponding to $r=1-2$. In that case the numerically induced effects can be comparable to or even larger than the turbulent stresses for typical discretisation methods such as central or upwind finite difference or finite volume methods. Thereby, the computational large-eddy closure contains an important contribution, which is sensitive to the adopted spatial discretisation.

Central to a framework for assessing the error-behaviour in an actual large-eddy implementation is the evaluation of the total simulation error and its decomposition into numerical and modelling components. By appropriately comparing large-eddy and direct simulation predictions, the total simulation-error can be quantified (Geurts, Froehlich, 2002). Recently, a database of both direct and large-eddy simulations of decaying homogeneous, isotropic turbulence was analysed at two different Reynolds numbers (Meyers, Geurts, Baelmans, 2003). In particular, for the Smagorinsky eddy-viscosity model combined with second order finite volume discretisation, the dependence of modelling - and numerical errors on simulation parameters was discussed. The interaction between these two basic sources of error was shown to lead to their partial cancellation for several flow properties such as kinetic energy and velocity skewness.

In this paper we review the interacting error-dynamics in terms of a so-called error-landscape, which provides a concise visualisation of the induced errors. The 'optimal refinement strategy' that can be extracted from such a landscape, yields minimal total simulation error at given computational effort. Compared to the optimal refinement strategy, the error induced by the dynamic eddy-viscosity model (Germano et al., 1991) at different resolutions, is about a factor two larger. However, the rate by which the error reduces with increased resolution was shown to be quite strong, particularly at high resolutions (Meyers, Geurts, Baelmans, 2005). To compensate for the remaining high error-levels at coarse resolutions, a new successive inverse polynomial interpolation (SIPI) procedure was proposed (Geurts, 2006). This allows approximating efficiently the optimal Smagorinsky constant at given resolution. As point of reference, the procedure starts from predictions using either no subgrid model at all or the dynamic eddy-viscosity model at a given spatial resolution. The proposed iterative procedure rapidly converges toward the optimal model-parameter. The computational overhead of this procedure is well justified by the increased accuracy.

The organisation of this paper is as follows. In section 2 we introduce a database analysis of large-eddy simulation of homogeneous decaying turbulence in a 'Smagorinsky fluid'. An exhaustive database-approach is rather expensive, but 
allows identifying the optimal refinement strategy. To achieve more practical error-reduction approaches, the degree of optimality of the dynamic procedure is discussed. Further improvements in accuracy may be achieved by directly approximating the optimal model parameters at given resolution. The SIPI iteration is discussed in section 4. Concluding remarks are collected in section 5 .

\section{Database approach to interacting errors}

In this section we review the database approach for LES errors that gives an overview of the total simulation errors. This methodology was recently adopted to assess simulation errors in homogeneous turbulence (Meyers, Geurts, Baelmans, 2003). We illustrate the effect of partial error-cancellation. Throughout, we adopt the classical filtering approach to large-eddy simulation, and consider subgrid closure based on the classical Smagorinsky model (Smagorinsky, 1963), or on the dynamic eddy-viscosity model (Germano et al., 1991). Excellent text-books exist that review the filtering approach (Pope, 2000; Sagaut, 2001; Geurts, 2003).

We focus on the error-landscape associated with the Smagorinsky model and identify the so-called optimal refinement strategy. At given spatial resolution, the optimal refinement strategy corresponds to the value of the Smagorinsky parameter at which the total error is minimal. This refinement strategy also allows interpreting the degree of optimality of the popular dynamic eddy-viscosity model (Germano et al., 1991). In fact, this subgrid model will be shown to display too high levels of eddy-viscosity and yields significant errors at coarse resolutions. However, at higher resolutions, the dynamic modelling provides a strong reduction of the error-levels.

The database approach provides an 'experimental' quantification of the errors that arise in large-eddy simulation. In particular, it allows a detailed decomposition of the total error in a numerical - and a subgrid modelling contribution. This clarifies which effects form the dominant limitations for the overall accuracy of large-eddy simulations, and under what computational settings and flow-conditions this identification applies. An extensive large-eddy simulation database of homogeneous decaying turbulence was generated at a variety of resolutions and filter-widths using grids with $N$ by $N$ by $N$ cells where $N$ is up to 128 . Initial conditions at Taylor Reynolds numbers 50 and 100 were adopted. For the direct simulation $N=384$ or $N=512$ was used and the level of convergence for various flow quantities was established.

In terms of an explicit filter it is straightforward to define the different error contributions in a large-eddy simulation. We restrict ourselves to the resolved kinetic energy $E(t)$, which is a volume average of the velocity product $(\mathbf{u}, \mathbf{u})$. To quantify the error-behaviour, we introduce some basic notation. The total error in $E$ resulting from a 'Smagorinsky-fluid' is concisely expressed as function of the so-called 'Smagorinsky length-scale', i.e., the product of the filter-width $\Delta$ and the Smagorinsky parameter. The Smagorinsky subgrid resolution $\xi$ is the ratio 
between this Smagorinsky length and the grid-spacing $h=1 / N$. Finally, the relative error $\delta$ is defined in terms of the difference between the filtered DNS result and the actual LES prediction. Note that this is a convenient choice, adhered to in this paper - in case DNS data are not available, alternative error-measures can be considered, e.g., based on experimental data, external constraints or concepts of 'expected' smoothness of the numerical solution, e.g., giving a high error-weight to artificial grid-oscillations.

In the sequel we express the total error behaviour in terms of the Smagorinsky subgrid resolution $\xi$. This error can be further decomposed into a contribution due to the discretisation and one due to the subgrid modelling. To quantify these measures for the simulation error a direct numerical simulation and several largeeddy simulations at different Smagorinsky lengths and spatial resolutions are required. These data form the heart of the database approach. A central role is played by the grid-independent prediction of the decay of the resolved kinetic energy $E$ obtained with the Smagorinsky model at fixed Smagorinsky length and 'infinite' spatial resolution. This grid-independent large-eddy prediction provides a central point of reference in the error decomposition, next to the reference direct numerical simulation data. It is well approximated at resolutions where $\Delta / h=4-6$ (Geurts, Froehlich, 2002). This grid-independent large-eddy simulation should not be confused with an actual, more practical LES in which the ratio between Smagorinsky length and grid spacing is much smaller. Grid-independent LES certainly is not optimal in the sense of computational effort - its accuracy reflects the accuracy of the subgrid model and allows separate reasoning about which procedure for subgrid closure is physically consistent.

The total relative error $\delta$ is time-dependent. In order to arrive at a single number to characterise the error in any specific large-eddy simulation we introduce as measure the time-average square norm of the difference between the filtered DNS result and the LES prediction (Geurts, Froehlich, 2002). The error-behaviour can conveniently be described in terms of the 'error-landscape'. For the Smagorinsky fluid the error-landscape can be expressed using the subgrid resolution of the Smagorinsky length $\xi$ and the spatial resolution $N$ as basic parameters. Each combination of $\xi$ and $N$ corresponds to a single large-eddy simulation. The error landscape is formed by contours of the error-measure on the $(\xi, N)$ plane, and can be constructed from systematic variation of the simulation parameters. It provides the 'optimal refinement strategy', which identifies the optimal values of simulation parameters that leads to the smallest total error at a particular resolution.

The direct numerical simulation data and the grid-independent large-eddy predictions allow full separation of the modelling and discretisation contributions to the total error. In figure 1 a decomposition is collected in which we consider simulations at constant Smagorinsky length $h \xi$ and various resolutions. In this case we observe that a higher spatial resolution may yield a larger total error. In fact, the discretisation error effect decreases with increasing resolution and the 
total error approaches the modelling error. However, on the coarser grids, this modelling error is itself larger than the total error. On coarse grids the comparably large discretisation error effects partially cancel the modelling error effects.

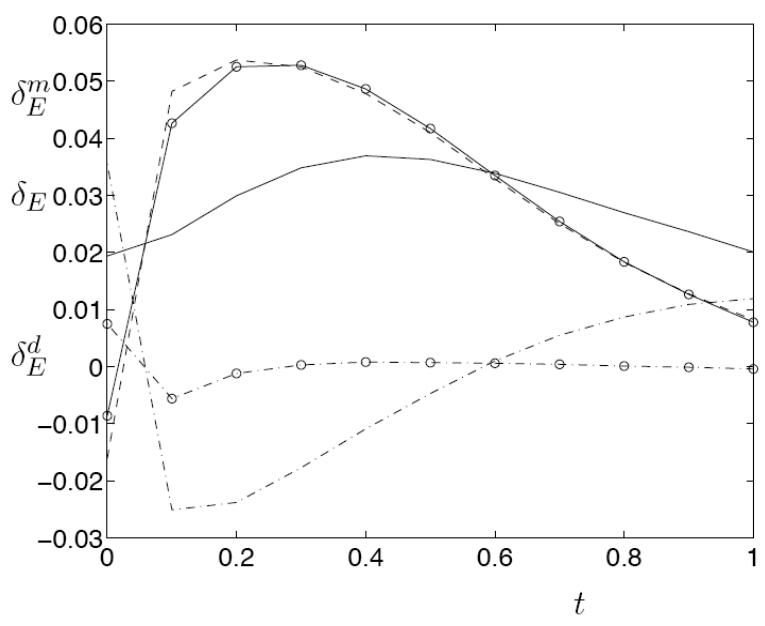

Figure 1 Error decomposition at Smagorinsky length 0.00625 for resolution $N=32$ (no symbol) and $N=64$ (o) displaying total error with solid curves, modelling error with dashed curves and discretisation error with dash-dotted curves.

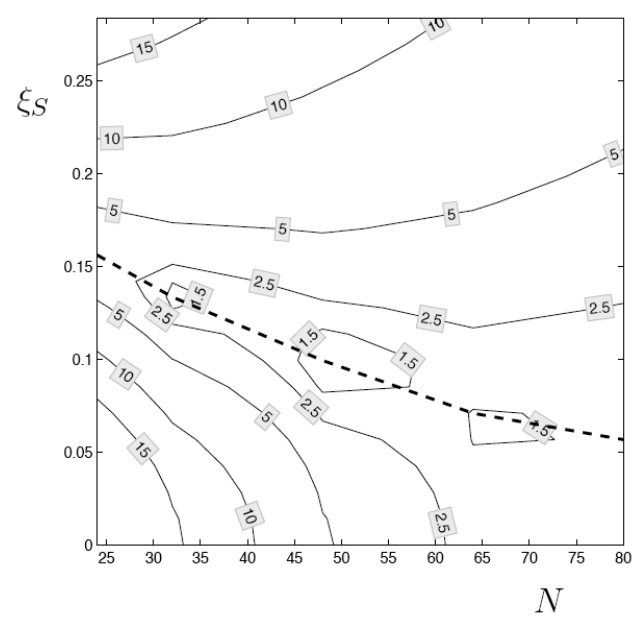

Figure 2 Error-landscape based on the time-integrated L2 norm of the kinetic energy differences at initial Taylor Reynolds number 100. The label on the contours refers to the relative error in $\%$. The thick dashed line corresponds to the optimal refinement strategy. 
Collecting the total errors as arise at various parameters settings in the $(\xi, N)$ plane, produces an overview as shown in the error-landscape in figure 2. From these contour plots the 'optimal refinement strategy' can be determined straightforwardly. At fixed, coarse resolutions, we observe a fairly sharp increase in the total simulation error in case the Smagorinsky parameter is below the optimal trajectory, while a slightly more gradual increase is observed in case $h \xi$ is larger than optimal.

The degree of optimality of the famous dynamic model (Germano et al., 1991) may readily be interpreted in relation to the error-landscape of the Smagorinsky fluid. In fact, the dynamic procedure gives rise to a self-induced length-scale, which can directly be translated into a 'dynamic refinement trajectory' in the error-landscape. Of particular importance is how this 'dynamic trajectory' relates to the 'optimal refinement strategy'.
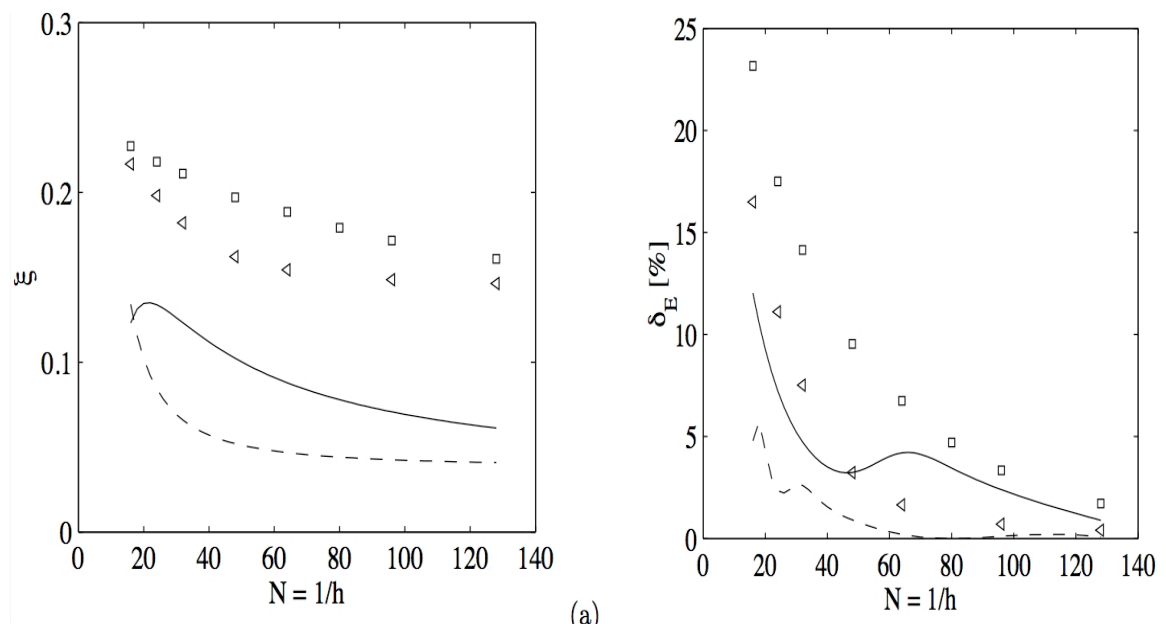

(a)

Figure 3 In (a) we show the optimal refinement strategies at Taylor Reynolds number 50 (dashed) and 100 (solid) compared to the dynamic refinement trajectories (triangles and squares respectively). In (b) the induced relative errors are shown as function of resolution.

In figure 3 we collected the dynamic refinement trajectories (Fig. 3a) for two different Taylor Reynolds numbers. The general trend in the dynamic length-scale is found to be similar to the optimal refinement strategy, although the dynamic refinement path is at somewhat too large values of the eddy-viscosity. In Fig. $3 b$ we observe that an increase in the resolution, with a test-filter width equal to $\Delta=2 h$, yields a strong error-reduction.

The errors along the dynamic refinement trajectories are about twice as large as the errors along the optimal refinement trajectory. An effective error-reduction 
arises from grid refinement. Particularly at higher resolutions a high rate of errorreduction with increased resolution is observed (further details may be found in (Meyers, Geurts, Baelmans, 2005). Conversely, at coarse resolutions the errors are still quite considerable and further improvement would be desired. This is the subject of the next section.

\section{Iterative approximation of the optimal Smagorinsky constant}

We propose an iterative procedure to improve predictions based on eddy-viscosity modelling at coarse subgrid resolutions. This iterative method is based on determining the minimum of the total simulation error. It allows efficient approximation of the optimal Smagorinsky constant at a particular resolution and leads to a significant error-reduction compared to the dynamic eddy-viscosity predictions.

To approximate the lowest total simulation error for the Smagorinsky fluid at given spatial resolution $N$ we may adapt the Smagorinsky constant iteratively. The first task is to obtain an interval $[a, c]$, which contains the optimal value for the resolution of the Smagorinsky length $\xi$. Subsequently, this interval will iteratively be reduced in size until an acceptable approximation of the minimal simulation error is achieved. As points of reference we start from the 'no-model' simulation which corresponds to $\xi=0=a$. This is the first simulation that is required in our iterative approach and it characterises the effects of discretization-error only. A second point of reference is obtained at fairly large $\xi$. A 'practical upper-bound' for $\xi$ may be obtained by taking $\xi(=c)$ to be the resolution of dynamic model length-scale. In the previous section we illustrated that this provides a reliable upper bound for the optimum. The evaluation of this error requires a second largeeddy simulation, now based on the dynamic eddy-viscosity model. Note that the dynamic eddy-viscosity model is used only to provide this upper-bound estimate; all other simulations employ the Smagorinsky model. The optimum is now bracketed by $[a, c]$.

In order to start the process of successive approximation of the optimal value for $\xi$ at the given resolution $N$, we evaluate the total error at an interior point of $[a, c]$. For this we select the mid-point $\xi=b=(a+c) / 2=c / 2$. The simulation at $\xi=b$ is the third large-eddy simulation in our iterative approach, which finalizes the initial set-up. Further improvements in $\xi$ may be obtained iteratively. In view of the high computational effort that is required to evaluate the 'error-function', only minimisation algorithms that do not rely on the explicit use of derivatives will be considered (see Brent, 1973). 


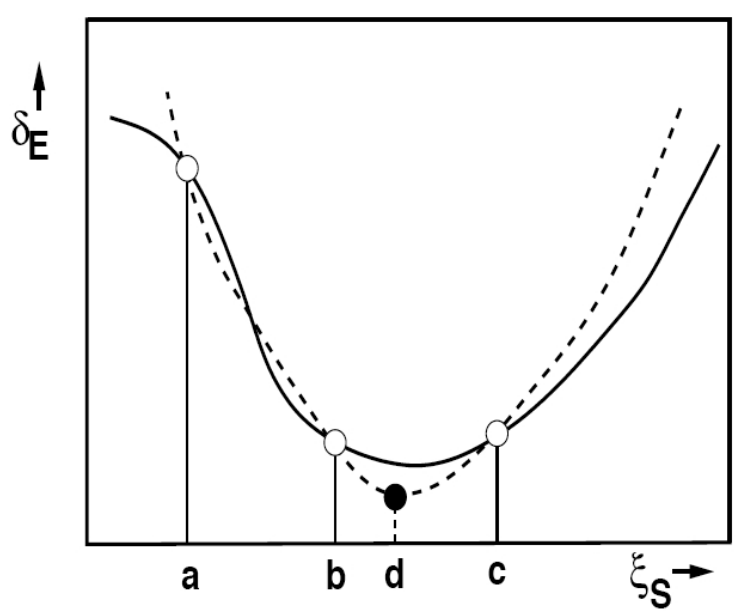

Figure 4 Successive inverse parabolic interpolation (SIPI) is illustrated, approximating the optimal resolution of the Smagorinsky length-scale $\xi$. The initial triplet $(a, b, c)$ defines an interpolating polynomial (dashed), whose minimum yields a next approximation $d$ at which a new large-eddy simulation should be performed.

Locally around its minimum we assume that a parabola may approximate the error-function. This motivates the use of successive inverse parabolic interpolation (SIPI) to obtain a next estimate for $\xi$. Referring to figure 4 we start by constructing an interpolating parabola through the original bracketing triplet. The location of the minimum of this parabola is at $d$. A new simulation is performed corresponding to $\xi=d$ and the total error can be obtained at this new parameter value. From this information a new bracketing triplet may be identified which, in turn, defines a new interpolating polynomial, and the process may be continued. Successive inverse parabolic interpolation and evaluation of the error-function leads to a sequence of bracketing triplets, which quite rapidly converges to the optimum (Geurts, 2006). If the error-function has a continuous second derivative, which is positive at the minimum, then convergence is super-linear (Brent, 1973). The application of this method to the Smagorinsky fluid is illustrated in figure 5. SIPI yields a rapid convergence. After about 4-6 large-eddy simulations the optimum is well approximated and a relative error of about $1-2 \%$ remains.

The computational overhead of the additional large-eddy simulations required for the iterations is well justified. This may be quantified by incorporating the scaling of CPU time with resolution, i.e., proportional to the fourth power of $N$ (Geurts, Froehlich, 2002). In fact, the completion of 4-6 simulations at resolution $N$ is comparable in cost to just one simulation at resolution $3 N / 2$. However, the reduction in the total simulation error arising from SIPI is typically much more pronounced than the error-reduction that may be achieved by increasing the resolution by $50 \%$ in each direction. For example, comparison of the optimal 
results at $N=32$ with results obtained with the dynamic model at $N=48$ shows that the error is reduced by a factor of about $4-5$ as a result of applying SIPI.

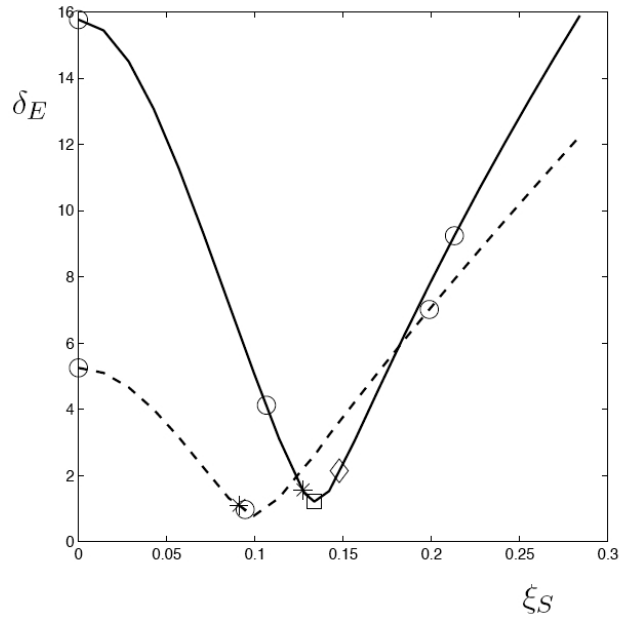

Figure 5 Application of SIPI to the total simulation error at Taylor-Reynolds number 100 and $N=32$ (solid) and $N=48$ (dashed). The initial triplet is indicated with open circles, the first iterand with *, the second with diamond and the third with square.

The optimisation procedure described here provides a very simple illustration of the consequences of interacting errors. The error-reduction relies specifically on the structure of the error-landscape. Further developments are required to render this approach suitable for more complex applications and to allow for 'contextspecific' extensions to cases in which DNS data are not available. Here, we compared LES to available DNS data - this constitutes a rather academic setting. Moreover, we only considered deviations with respect to one flow property. In general, one encounters situations in which the accurate prediction of a collection of 'monitoring quantities' is desired. This requires simultaneous accuracy for each of these quantities and implies an appropriate weighing of individual errors as part of the total error-measure (Meyers, Sagaut, Geurts, 2006).

\section{Concluding Remarks}

In this paper we considered the modifications of the large-eddy closure problem arising from the spatial discretisation at coarse subgrid resolutions. Moreover, a new iterative method for approximating the optimal Smagorinsky constant at low resolutions was proposed and applied to homogeneous, decaying turbulence.

In case the sub-filter resolution $r=\Delta / h$ is low, the particular discretisation scheme that is adopted in the computational model, was shown to have a large dynamic 
effect. The difference between the actual computational stress-tensor and the turbulent stress-tensor may be expressed most directly in terms of the modified equation (Geurts, Van der Bos, 2005). Fourier analysis showed that for values as low as $r=1-2$ the induced numerical contribution is comparable to or even larger than the term that requires closure in large-eddy simulation. This was observed earlier in a posteriori analysis of turbulent mixing (Vreman, Geurts, Kuerten, 1996). In case $r>4$ it appears that the adverse dynamic consequences of the discretization errors can largely be neglected.

The basic modelling and discretisation errors were found to counteract, e.g., in predictions of the resolved kinetic energy. This leads to an intriguing paradox related to possible strategies that should be followed to further improve large-eddy predictions compared to some reference simulation. While it is tempting to think that a higher resolution, a better numerical method or a more precise subgrid model would always lead to improved accuracy of the predictions, the counteracting property of the errors can completely distort this impression. Rather, the total error arises from a balance between modelling - and discretisation errors and it is not an easy matter to predict a priori whether these errors will or will not counteract and what the magnitude of the individual error contributions is.

The optimal working conditions for large-eddy simulations may be inferred from an error-landscape. The use of optimal refinement strategies as a point of reference for the evaluation of the dynamic procedure was reviewed. The dynamic procedure was found to provide a build-in 'dynamic trajectory', which follows the main Reynolds-number and resolution trends seen in the 'optimal refinement strategy' relatively well. However, an over-prediction of the optimal resolution of the Smagorinsky length-scale is obtained, which was found to lead to errors about twice as high as the optimal errors.

To improve upon these shortcomings of the popular eddy-viscosity model, a new iterative optimisation procedure for the Smagorinsky constant was proposed. This procedure is based on successive inverse polynomial interpolation (SIPI) and yields strongly improved accuracy compared to predictions based on the dynamic eddy-viscosity model. Initially, this method requires a bracketing interval for which simulations without subgrid model and with the dynamic eddy-viscosity model were adopted. The computational overhead associated with this iterative procedure is well justified in view of the increased accuracy compared to the dynamic eddy-viscosity model. Without the optimisation, such error-level would require much higher resolutions and computational costs. Further analysis of this new iterative procedure is subject of ongoing research.

\section{Acknowledgement}

The author gratefully acknowledges stimulating discussions with Johan Meyers (KU Leuven) and Jochen Froehlich (Karlsruhe). Simulations were performed at 
the Netherlands supercomputing center, SARA, and made possible through grant SG-213 of the Dutch National Computing Foundation (NCF).

\section{References}

Geurts, B.J.(2003): Elements of direct and large eddy simulation. Edwards Publishing, Inc.

Sagaut, P. (2001): Large eddy simulation for incompressible flows; an introduction, Scientific Computation, Springer Verlag.

Meneveau, C., Katz, J (2000): Scale-invariance and turbulence models for large eddy simulation. Ann. Rev. Fluid Mech., 32, 1.

Lesieur, M., Metais, O. (1996): New Trends in Large-Eddy Simulations of Turbulence. Ann. Rev. Fluid Mech., 28, 45.

Rogallo, R.S., Moin, P. (1984): Numerical simulation of turbulent flows. Ann.Rev. Fl. Mech. 16, 99.

Salvetti, M.V., Beux, F. (1998): The effect of the numerical scheme on the subgrid scale term in large-eddy simulation. Phys. of Fluids, 10, 3020.

Geurts, B.J., Froehlich, J. (2002): A framework for predicting accuracy limitations in large eddy simulation. Phys. of Fluids, 14, L41.

Meyers, J., Geurts, B.J., Baelmans, M. (2003): Database analysis of errors in large eddy simulation. Phys. of Fluids, 15, 2740.

Geurts, B.J., Bos, F. van der (2005): Numerically induced high-pass dynamics in largeeddy simulation, Phys. of Fluids, 17125103.

Ghosal, S. (1996): An analysis of numerical errors in large-eddy simulations of turbulence. J. Comp. Phys. 125, 187.

Germano, M., Piomelli, U., Moin, P., Cabot, W.H. (1991): A dynamic subgrid-scale model. Phys. Fluids 3, 1760.

Pope, S.B. (2000): Turbulent flows. Cambridge University Press.

Meyers, J., Geurts, B.J., Baelmans, M. (2005): Optimality of the dynamic procedure for large-eddy simulation. Phys. Fluids 17, 045108.

Geurts, B.J., Meyers, J. (2006): Successive inverse polynomial interpolation to optimize Smagorinsky's model for large-eddy simulation of homogeneous turbulence, Physics of Fluids, 18, 118102

Smagorinsky, J. (1963): General circulation experiments with the primitive equations. Mon. Weather Rev. 91, 99.

Brent, R. (1973): Algorithms for Minimization without Derivatives. Englewood Cliffs N.J.: Prentice-Hall.

Meyers, J., Geurts, B.J., Sagaut, P. (2006): Optimal model parameters for multi-objective large-eddy simulatons, Phys. of Fluids, 18, 095103.

Vreman, A.W., Geurts, B.J., Kuerten, J.G.M. (1996): Comparison of numerical schemes in Large Eddy Simulation of the temporal mixing layer, Int.J.Num. Meth. in Fluids 22, 299. 